\title{
Preparation of robust polyamide microcapsules by interfacial polycondensation of $p$-phenylenediamine and sebacoyl chloride and plasticization with oleic acid
}

\author{
Natacha Rosa ${ }^{1}$, Gabriela V. Martins ${ }^{1}$, Margarida M.S.M. Bastos ${ }^{1}$, Joana R. Gois ${ }^{2}$, Jorge F.J. Coelho ${ }^{2}$, Juliana Marques ${ }^{3}$, \\ Carlos J. Tavares ${ }^{3}$, and Fernão D. Magalhães ${ }^{1}$ \\ ${ }^{1}$ LEPABE, Faculdade de Engenharia, Universidade do Porto, rua Dr. Roberto Frias, Portugal, ${ }^{2}$ Chemical Engineering Department, CEMUC, University \\ of Coimbra, rua Sílvio Lima, Coimbra, Portugal, and ${ }^{3}$ Centre of Physics, University of Minho, Azurém Campus, Guimarães, Portugal
}

\begin{abstract}
Microcapsules produced by interfacial polycondensation of p-phenylenediamine (PPD) and sebacoyl chloride (SC) were studied. The products were characterized in terms of morphology, mean diameter and effectiveness of dodecane encapsulation. The use of Tween 20 as dispersion stabilizer, in comparison with polyvinyl alcohol (PVA), reduced considerably the mean diameter of the microcapsules and originated smoother wall surfaces. When compared to ethylenediamine (EDA), microcapsules produced with PPD monomer were more rigid and brittle, prone to fracture during processing and ineffective retention of the core liquid. The use of diethylenetriamine (DETA) cross-linker in combination with PPD did not decrease capsule fragility. On the other hand, addition of a small fraction of oleic acid to the organic phase remarkably improved wall toughness and lead to successful encapsulation of the core-oil. Oleic acid is believed to act as a plasticizer. Its incorporation in the polymeric wall was demonstrated by FTIR and ${ }^{1} \mathrm{H}-\mathrm{NMR}$.
\end{abstract}

\section{Keywords}

Fragility, interfacial polymerization, mechanical stability, microencapsulation, smooth surface, surfactant

\section{History}

Received 11 July 2014

Revised 6 February 2015

Accepted 9 February 2015

Published online 8 June 2015

\section{Introduction}

Microencapsulation is used for various applications involving shielding or controlled release of an active substance, ranging from agriculture to pharmacology fields (Danicher et al., 2000; Zydowicz et al., 2001; Essawy and Tauer, 2010). Among the different techniques available for microcapsule production, interfacial polycondensation has received particular attention since it is a rapid and straight-forward procedure (Wittbecker and Morgan, 1996; Wagh et al., 2009). Studies on this technique started in the early 1950's (Berezkin and Khokhlov, 2006). The process can be described as soft-templating of emulsion droplets (Essawy and Tauer, 2010). Most often encapsulation of an organic compound is intended, and an oil-in-water emulsion is produced as starting point. However, encapsulation of hydrophilic molecules is also possible (Zydowicz et al., 2001).

A common implementation of encapsulation by interfacial polymerization consists on the formation of polyamide shells by reaction of an acyl chloride with a polyfunctional amine. The first monomer is dissolved in a dispersed organic phase, while the amine is present in the continuous aqueous phase (Freger, 2003; Benita, 2005; Mittal, 2013). It is commonly accepted that the polycondensation reaction takes place at the organic side of the interface, due to the negligible solubility of acyl chlorides in water and the fairly good solubility of amines in organic solvents (Freger, 2003).

Address for correspondence: F.D. Magalhães, LEPABE, Faculdade de Engenharia, Universidade do Porto, rua Dr. Roberto Frias, 4200-465 Porto, Portugal. Tel: +351 225081601. E-mail: fdmagalh@fe.up.pt
A common problem associated with polyamide microcapsules is fragility. These structures are often unable to withstand processing conditions like filtering, washing and drying for obtaining powders, or high-shear dispersion in viscous liquids for incorporation in coatings (Latnikova, 2012). Some strategies mentioned in the literature to improve the robustness of microcapsules are double-wall formation (Caruso et al., 2010), addition of inorganic nano-fillers (Chuanjie and Xiaodong, 2009; Wu et al., 2014) and increase of shell thickness (Li et al., 2013). However, the application of some of these approaches is unfeasible in interfacial polymerization, or implies complex multi-step procedures. Latnikova (2012) suggested that introducing an internal plasticization effect by using "soft" monomers with large hydrocarbon chains should improve mechanical stability of polyamide microcapsules, but the strategy was not tested.

The present work reports the use of $p$-phenylenediamine (PPD) as hydrophilic monomer for production of polyamide microcapsules by interfacial polycondensation with sebacoyl chloride (SC). To the best of our knowledge, this is the first report on the combination of these two monomers for microencapsulation. The effect of different operating conditions and the importance of the type of stabilizer on the size, size distribution and surface morphology of the microcapsules are discussed. Perfectly spherical shells with very smooth external surfaces can be obtained, but these exhibit low mechanical stability. Strategies to overcome this fragility were studied and good results were obtained adding oleic acid to the dispersed organic phase. This oil-soluble surfactant migrates to the water-oil interface and was incorporated in the shell material during interfacial polymerization. The plasticizing 
effect of oleic acid, reported here for the first time, provides a novel and straightforward strategy for improving the mechanical stability of microcapsules produced by interfacial polymerization, with potential use in combination with other monomers.

\section{Experimental}

\section{Materials}

All chemicals listed below were purchased from Sigma-Aldrich (St. Louis, MO), except for oleic acid, which was supplied by Acros-organics (Geel, Belgium). Capsule shell monomers: sebacoyl chloride (SC; 99\%), ethylenediamine (EDA; 99\%), p-phenylenediamine (PPD; 99\%), diethylenetriamine (DETA; 99\%). Capsule core material: dodecane (anhydrous, 99\%). Dispersion stabilizers: poly(vinyl alcohol) (PVA, $\mathrm{M}_{\mathrm{w}}=13000$ 23000, 98\% hydrolysed) and polyoxyethylene sorbitan monolaurate (commonly known as Tween 20). Capsule shell plasticizer: oleic acid (97\%).

\section{Methods}

\section{Production of microcapsules}

Microencapsulation of dodecane was carried out in a $100 \mathrm{~mL}$ glass flask equipped with a mechanical stirrer (Cowles impeller, $25 \mathrm{~mm}$ diameter). The oil solution was prepared by mixing $1 \mathrm{~mL}$ of dodecane and $0.2 \mathrm{~mL}$ of $\mathrm{SC}$ at room temperature. The dispersion of oleic acid in the organic phase was performed under ultrasonication. The organic mixture was added dropwise under vigorous stirring into $25 \mathrm{~mL}$ of aqueous solution containing dispersion stabilizers. These were PVA (at a concentration of 2 wt.\%), a mixture of PVA (2 wt.\%) and Tween 20 (1 wt.\%), or Tween 20 (1 wt.\%) alone. Once this addition was terminated, the stirring speed was decreased, as discussed later, and $25 \mathrm{~mL}$ of amine solution was slowly added to the reactor during a period of $30 \mathrm{~min}$. The excess amine was used to neutralize hydrochloric acid produced. The reaction was continued for $30 \mathrm{~min}$ after amine addition to ensure complete formation of the polymeric membrane. Finally, the microcapsules were isolated by filtration and washed with distilled water to remove unreacted amine.

\section{Characterization of microcapsules}

Samples of microcapsules dispersions were collected after synthesis and observed in an Olympus IX 51 inverted optical microscope (Tokyo, Japan). Digital images were recorded and analysed in ImageJ software (National Institutes of Health, Bethesda, MD). At least 100 microcapsule diameters were measured for calculation of the mean diameters and standard deviations in each production batch. Optical microscopy images were also used for analysing the morphology of the microcapsules in the liquid dispersion.

The size and morphology of dried microcapsules were examined by Scanning Electron Microscopy (SEM), using a FEI Quanta 400FEG ESEM / EDAX Genesis X4M (Hillsboro, OR) with acceleration voltage of $3 \mathrm{kV}$, at Centro de Materiais da Universidade do Porto. For this purpose, dried capsules were sputtered with a thin layer $(10 \mathrm{~nm})$ of gold-palladium. The samples were analysed under vacuum. In order to evaluate the percentage of damaged microcapsules, square regions of SEM images collected at 200 magnification, containing at least 60 capsules, were randomly selected and the number of damaged and undamaged microcapsules counted. The mean and standard deviation values were then computed based on the results of at least three such samples.

The composition of the shell polymer was analysed by Fourier Transform Infrared Spectroscopy (FTIR). Samples were previously crushed, washed and dried for $12 \mathrm{~h}$. The FTIR spectra were recorded at room temperature using an ABB-Bomem spectrometer (Quebec City, Canada) in transmittance mode. Each spectrum was an average of 128 scans taken with $4 \mathrm{~cm}^{-1}$ resolution in the $4000-650 \mathrm{~cm}^{-1}$ range. The incorporation of oleic acid in the microcapsules was investigated by using the integration of the peaks height (in software Win-Bomem Easy, Version 3.04, Salem, NH). The absorbance of characteristic bands was compared to reference bands.

${ }^{1} \mathrm{H}-\mathrm{NMR}$ spectra were acquired on a Bruker Avance 400 spectrometer (Billerica, MA) operating at a frequency of 300 $\mathrm{MHz}$. The spectra were recorded at room temperature with 1-2\% $(\mathrm{w} / \mathrm{v})$ sample solutions in dimethylsulfoxide (DMSO- $\left.d_{6}\right)$. PPD microcapsules prepared with oleic acid were crushed, washed and dried for removal of the organic phase and then dissolved in DMSO- $d_{6}$. Chemical shifts were reported in parts per million $(\mathrm{ppm}, \delta$ ).

Thermogravimetric Analysis (TGA) was carried out on a STA Netzsch Jupiter 449 F3 (Selb, Germany). The weight of the samples was $2-5 \mathrm{mg}$. They were heated at $10^{\circ} \mathrm{C} / \mathrm{min}$ from $25^{\circ} \mathrm{C}$ to $600{ }^{\circ} \mathrm{C}$ under constant nitrogen flow.

\section{Results and discussion}

\section{Selection of operating conditions}

Encapsulation by interfacial polycondensation involves two main stages: (1) emulsification of the organic phase, containing lipophilic monomer, in aqueous medium, and (2) addition of hydrophilic monomer. The first stage determines the size of the microcapsules, since an apparent steady state size distribution is established as a result of oil phase rupture, due to mechanical shearing, versus droplet coalescence, caused by collisions and surface tension forces (Zydowicz et al., 2001; Soto-Portas et al., 2003). The second step is responsible for the formation of the polymeric membrane at the water/oil interface. This preliminary study focused on the effect of varying stirring speed and time on the emulsification step. A short length aliphatic diamine (EDA) was used as a hydrophilic monomer and PVA (2\% concentration in water) as a stabilizer (Hong and Park, 1999; Chen et al., 2012). Sebacoyl chloride was the lipophilic monomer adopted throughout the entire work. Another acyl chloride commonly used in microencapsulation by interfacial polymerization is terephthaloyl chloride (Essawy and Tauer, 2011). However, its insolubility in the organic phase (dodecane) did not allow its use. An amine:acyl chloride ratio of 10:1 was used. At the end of the polycondensation, complete creaming of the capsules was visible, while the aqueous phase showed no turbidity. The microcapsules were collected from the creamed phase and observed by optical microscopy immediately after preparation.

Table 1 summarizes the results of varying the impeller stirring speed during the emulsification process. These suggest a significant decrease in the mean diameter of the microcapsules when increasing the stirring speed: from $545 \mu \mathrm{m}$ at $400 \mathrm{rpm}$ to $55 \mu \mathrm{m}$ at

Table 1. Mean diameter, absolute and relative span values of EDA+SC microcapsules produced with different mechanical stirring speeds during three minutes in the emulsification stage.

\begin{tabular}{lccc}
\hline $\begin{array}{l}\text { Stirring speed } \\
(\mathrm{rpm})\end{array}$ & $\begin{array}{c}\text { Mean microcapsule } \\
\text { diameter }(\mu \mathrm{m})\end{array}$ & $\begin{array}{c}\text { Absolute } \\
\text { span }(\mu \mathrm{m})\end{array}$ & $\begin{array}{c}\text { Relative } \\
\text { span }\end{array}$ \\
\hline 400 & 545 & 375 & 0.83 \\
1200 & 153 & 137 & 1.1 \\
1700 & 55 & 60 & 1.3 \\
\hline
\end{tabular}

Mean diameter values are based on number of particles and were computed from optical microscopy images. 


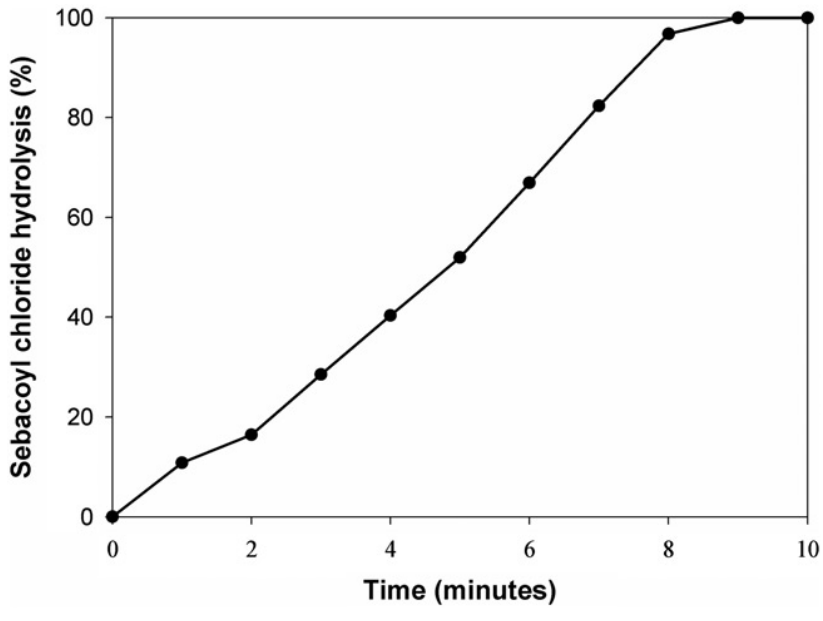

Figure 1. Percentage of SC hydrolyzed with time at constant emulsion stirring speed of $1200 \mathrm{rpm}$. Results refer to a single experiment.

$1700 \mathrm{rpm}$. The relative spans computed for the three particle size distributions also increase, which is in qualitative agreement with those reported by Mahabadi et al. (1996). An impeller speed of $1200 \mathrm{rpm}$ was used in all experiments performed subsequently. This value provided a relatively small microcapsule mean diameters with a low coefficient of variation.

Hydrolysis of acyl chloride is a side reaction that tends to decrease the amount of reactive lipophilic monomer available for interfacial membrane formation and consequently reduce polymer yield. The hydrolysis leads to formation of hydrochloric acid and, if no base is added for neutralization, a decrease in the $\mathrm{pH}$ of the aqueous phase is observed (Soto-Portas et al., 2003). The percentage of SC hydrolysed along the emulsification stage can therefore be stoichiometrically determined from $\mathrm{pH}$ measurements with time, if no neutralizing amine is added. Figure 1 shows that the computed degree of SC hydrolysis, at a constant emulsion mechanical stirring of $1200 \mathrm{rpm}$, increases almost linearly with time. After approximately eight minutes stirring there is virtually no reactive lipophilic monomer available. Although SC, due to its long alkyl chain, is considered to be one of the less sensitive monomers to hydrolysis (Soto-Portas et al., 2003), these data shows that formation of the polyamide microcapsules may be jeopardized if the emulsification time is not controlled. Also, other factors such as the type and geometry of impeller and dimensions of the reaction vessel can have an impact on the rate of hydrolysis (Yan et al., 1994; Zhang et al., 1995).

On the other hand, emulsification time may also have an impact on droplet size and hence on the final microcapsule diameter. However, it was observed that for stirring times longer than three minutes microcapsule sizes remained essentially unchanged. An emulsification time of three minutes was therefore chosen for the remaining work.

During the second stage, the polyamide capsule is formed as the amine diffuses into the oil droplets and reacts with lipophilic monomer. The hydrochloric acid produced in the polycondensation reaction can be neutralized by an added alkali or excess amine monomer (Arshady, 1989). As described in the methods section, the second approach was followed in this work, similarly to other studies (Zydowicz et al., 2001; Soto-Portas et al., 2003), using a $\mathrm{NH}_{2} / \mathrm{COCl}$ molar ratio equal to 10 . The impeller stirring rate during amine addition was observed to affect capsule integrity. Ruptured microcapsules were visible in the aqueous medium by optical microscopy for stirring speeds above $800 \mathrm{rpm}$, indicating that shear rate was too high for the polymeric membranes, at least during the initial stages of formation. A stirring speed of $400 \mathrm{rpm}$ was adopted for this stage.
Table 2. Amine and surfactant combinations.

\begin{tabular}{lcl}
\hline Formulation & Amine & \multicolumn{1}{c}{ Surfactant } \\
\hline A & EDA & $2 \%$ PVA \\
B & & $2 \%$ PVA $+1 \%$ Tween 20 \\
C & $1 \%$ Tween 20 \\
D & PPD & $2 \%$ PVA \\
E & & $2 \%$ PVA $+1 \%$ Tween 20 \\
F & & $1 \%$ Tween 20
\end{tabular}

Surfactant concentrations are presented as weight percentage in solution.

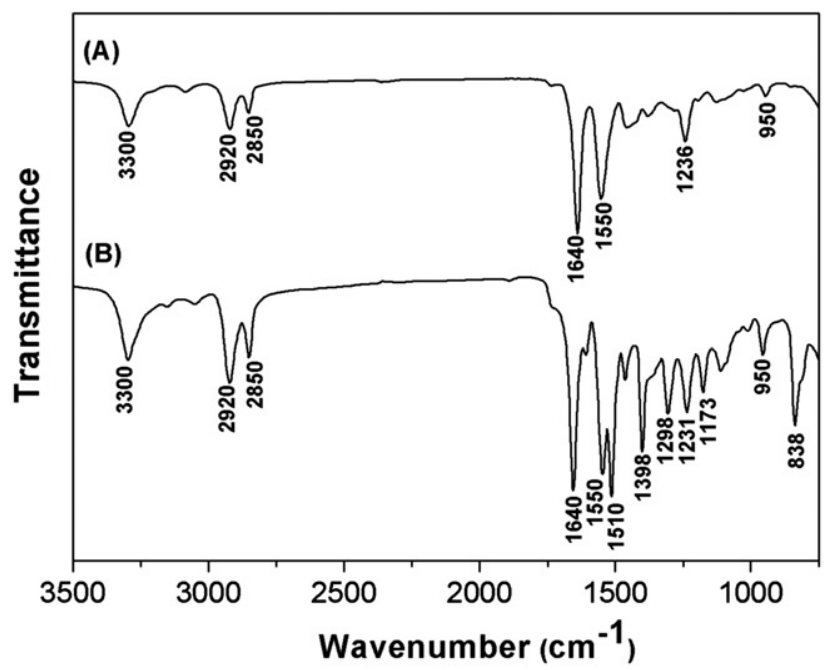

Figure 2. FTIR spectra of microcapsules obtained from EDA (curve A) and PPD (curve B).

In summary, the chosen operating conditions consisted on stirring at $1200 \mathrm{rpm}$ for three minutes in the emulsification stage, followed by amine addition at a stirring speed of $400 \mathrm{rpm}$.

\section{Effect of hydrophilic monomer and stabilizer type}

The formulations tested with different diamines (EDA and PPD) and surfactant combinations (PVA and Tween 20) are presented in Table 2. The amine/acyl chloride ratios were kept constant and equal to 10 . The two amines tested are short length bifunctional hydrophilic monomers, one with an aliphatic structure - EDA and the other with an aromatic ring - PPD. To the best of our knowledge, there is no previous report on the use of PPD on microencapsulation by interfacial polymerization. PVA is commonly used in the literature as the sole stabilizer in this type of encapsulation process (Soto-Portas et al., 2003; Dubey, 2009), but ionic or non-ionic surfactants have also been reported (Scarfatoet al., 2007; Chen et al., 2012).

Figure 2 shows the FTIR spectra for EDA (A) and PPD (B) microcapsules. Both spectra show the characteristic band for $\mathrm{N}-\mathrm{H}$ stretching of amides at around $3300 \mathrm{~cm}^{-1}$. In addition, two bands are visible at 1640 and $1550 \mathrm{~cm}^{-1}$, which are assigned to polyamide I and II, proving that polyamide wall has been successfully produced. $\mathrm{C}-\mathrm{H}$ stretching vibration for $\mathrm{CH}_{2}$ and $\mathrm{CH}_{3}$ groups are found at 2850 and $2920 \mathrm{~cm}^{-1}$. Also, the absence of the vibrational band corresponding to the group $\mathrm{R}-\mathrm{COCl}$ from the monomer SC $\left(\sim 1800 \mathrm{~cm}^{-1}\right)$ suggests that it was completely consumed, or at least hydrolyzed. Only spectrum (B) presents peaks around $1510 \mathrm{~cm}^{-1}$ and $838 \mathrm{~cm}^{-1}$, which are assigned to $\mathrm{C}=\mathrm{C}$ stretching and $\mathrm{C}-\mathrm{H}$ deformation, respectively, of the aromatic ring in PPD. 

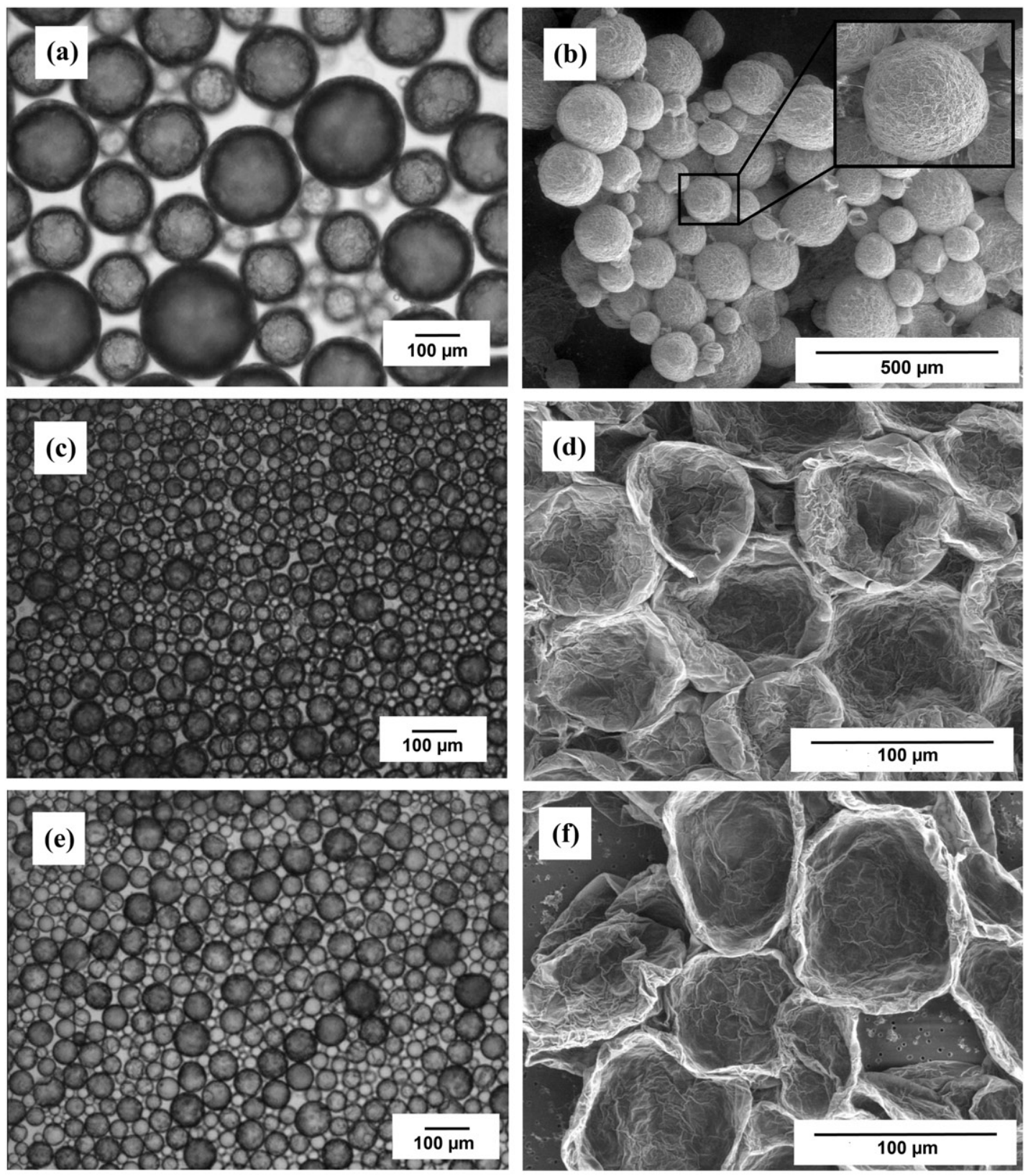

Figure 3. Morphological aspect of the microcapsules synthesized using EDA, by optical microscopy (a, c, e) and scanning electron microscopy (b, d, f). The stabilizer system used was: $2 \%$ PVA (a, b), 2\% PVA $+1 \%$ Tween 20 (c, d) and $1 \%$ Tween 20 (e, f), respectively.

Optical microscopy and SEM images of the microcapsules obtained from EDA and PPD are presented in Figures 3 and 4, respectively. For the microcapsules produced with EDA, use of Tween 20, in combination with PVA or alone (Figures $3 \mathrm{c}$ and e), reduces the mean diameters considerably, from about $110 \mu \mathrm{m}$ to $60 \mu \mathrm{m}$, when comparing to the microcapsules stabilized with PVA alone (Figure 3a). As expected, decreasing interfacial tension causes formation of smaller oil-in-water droplets. A similar effect is seen with PPD microcapsules, comparing Figure 4(a) to Figure 4(c and e). Unexpectedly, however, introduction of Tween 20 also caused a change in surface morphology. When PVA was used alone, microcapsules produced with either EDA or PPD showed a rough surface. On the other hand, use of Tween 20, alone or combined with PVA, yielded capsules with very smooth surfaces. Note that the wrinkles observed in Figure 3(d) and (f) are due to collapse of the microcapsules when under vacuum in the SEM chamber, as often observed in this type of systems (Essawy and Tauer, 2010). The fact that, when using Tween 20, EDA microcapsules are collapsed while PPD microcapsules remain spherical is a consequence of the benzenic ring in the latter, yielding a more rigid polyamide wall than aliphatic EDA. However, this higher rigidity seems to be associated with higher fragility, as shown by the abundant presence of cracked and fractured structures (Figure $4 \mathrm{~d}$ and $\mathrm{f}$ ). This damage probably occurs during processing of the capsules (washing and drying). The corresponding optical microscopy images (Figure 4c and e) show apparently well-formed and intact spheres in the original aqueous medium, after synthesis.

Figure 5 presents a SEM cross-sectional image of a microcapsule wall obtained with formulation E (PPD and 2\% PVA $+1 \%$ Tween 20). A very smooth nonporous external wall and an irregular internal surface are visible. In interfacial polymerization, the reaction occurs at the organic phase side (Dubey, 2009). The diamine diffuses from the aqueous phase, across the forming capsule skin, and then reacts with the acyl chloride end groups. This inward growth of the polymeric wall resulted in the formation of an irregular morphology (Song et al., 2005). The observed smoothness of the external surface, obtained only in the 

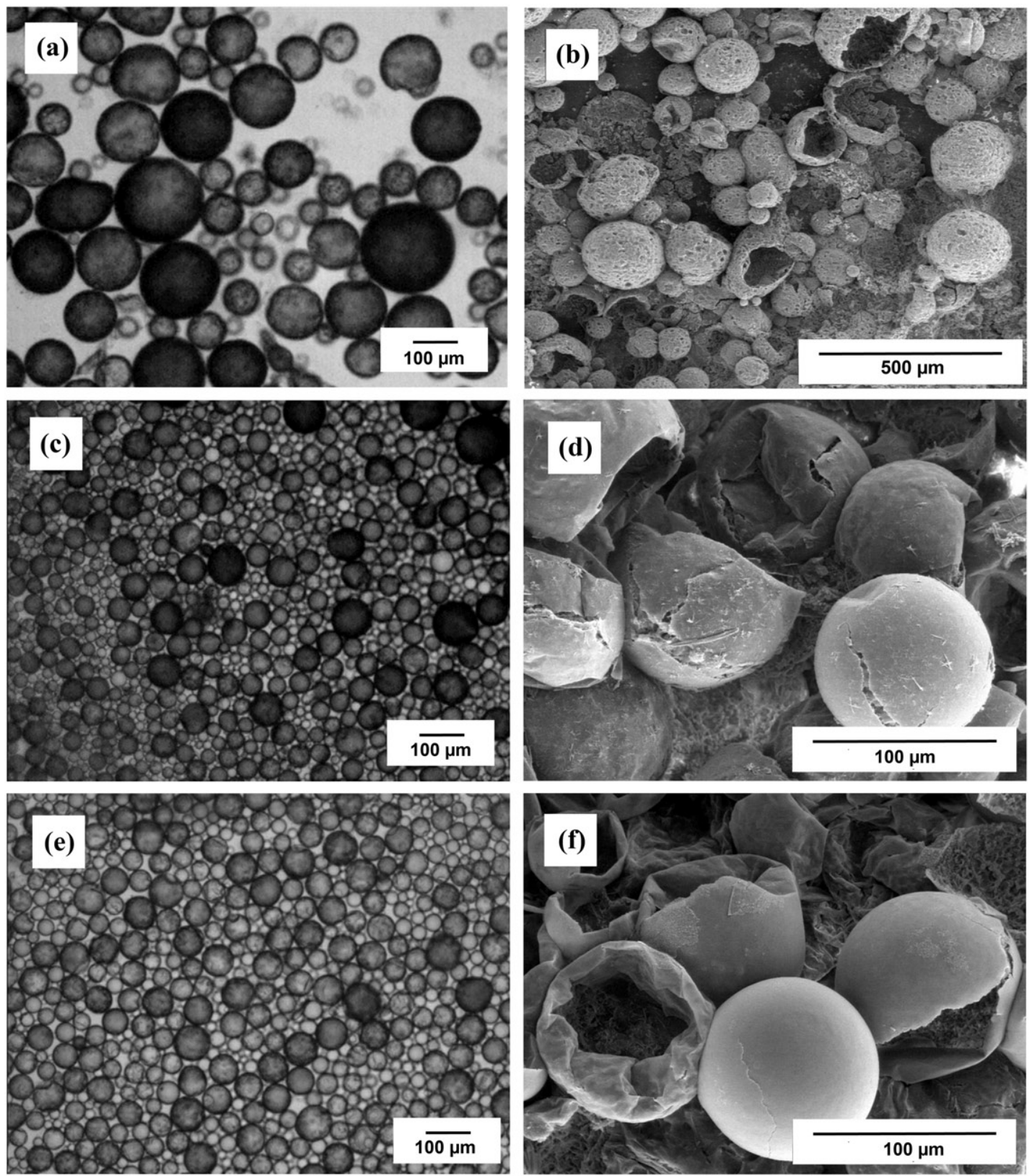

Figure 4. Morphological aspect of the microcapsules synthesized using PPD, by optical microscopy (a, c, e) and scanning electron microscopy (b, d, f). The stabilizer system used was: $2 \%$ PVA (a, b), 2\% PVA + 1\% Tween 20 (c, d) and 1\% Tween 20 (e, f), respectively.

presence of Tween 20, can be rationalized taking into account the interaction of both stabilizers in the surface. PVA is not actually a surfactant but a protective colloid. Some portions of the molecule adsorb at the aqueous/organic interface and the chain assumes a folded conformation that provides steric stabilization against coalescence or agglomeration (Goel and Rani, 2012) (Figure 6a). In addition, PVA is capable of establishing hydrogen bonds with the growing polyamide, and interpenetration may occur. Tween 20 , on the other hand, has an amphiphile structure that promotes head/tail orientation at the aqueous/organic interface (Figure 6b). The different conformations that the two stabilizers assume at the interface may therefore provide a rationale for the distinct external capsule morphologies. The fact that the combination of Tween 20 with PVA originates the same result as Tween 20 alone can be explained considering that Tween 20 adsorbs preferentially at the interface, forcing PVA to remain in the aqueous phase. As a result of this competitive effect, PVA ends up not playing a role on the stabilization of the organic phase and, thus, on the morphology of the microcapsules.
The thermal stability of previously washed and dried shells, obtained from EDA and PPD microcapsules, was investigated by thermogravimetry and is shown in Figure 7. As expected, the PPD-based microcapsules degrade at a significantly higher temperature and yield higher amount of stable char residue at $600{ }^{\circ} \mathrm{C}$, due to the presence of aromatic rings in the polyamide chain.

\section{Effect of cross-linker and plasticizer addition}

Use of PPD as co-monomer for microcapsule formation is an interesting approach. As characteristic of polyamides obtained from aromatic instead of aliphatic amines, PPD has the potential to provide higher resistance and form a less permeable membrane (Soto-Portas et al., 2003), which can be advantageous for some applications. However, the observed brittleness of the capsule walls is a serious disadvantage. Addition of DETA, a trifunctional amine, often used as cross-linker in interfacial polymerization (Mahabadi et al., 1996; Danicher et al., 2000), 


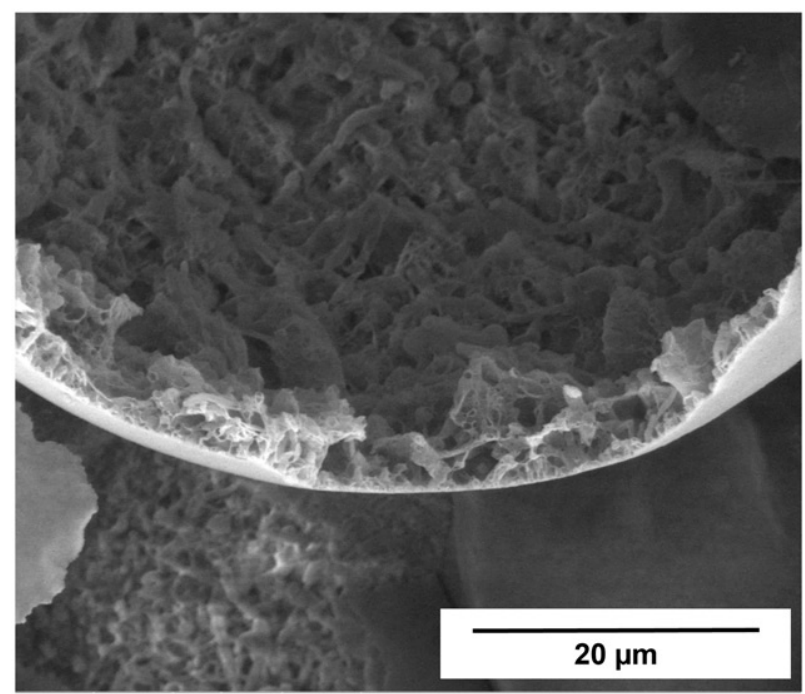

Figure 5. Scanning electron micrograph image of formulation E (PPD with $2 \%$ PVA $+1 \%$ Tween 20 ) microcapsule interior wall.
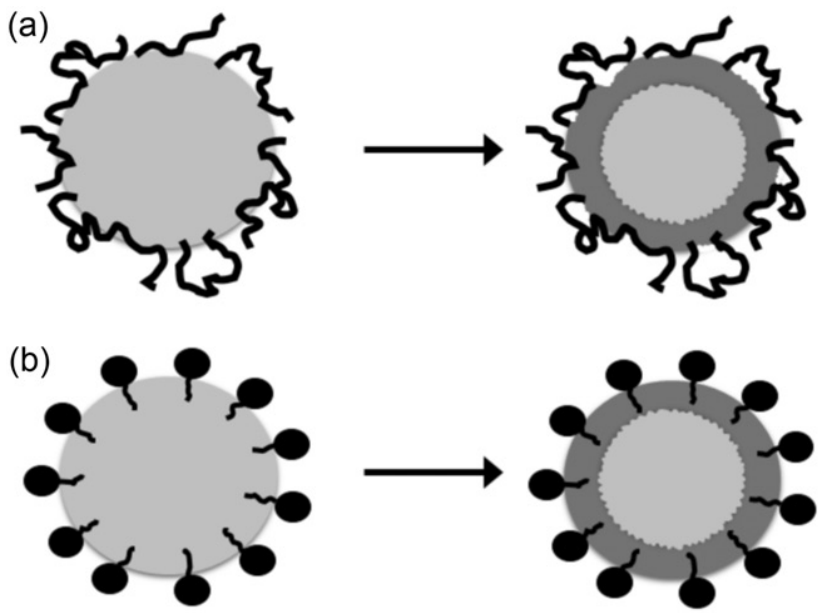

Figure 6. Schematic representation of stabilizers conformation at the interface: PVA (a) and Tween 20 (b).

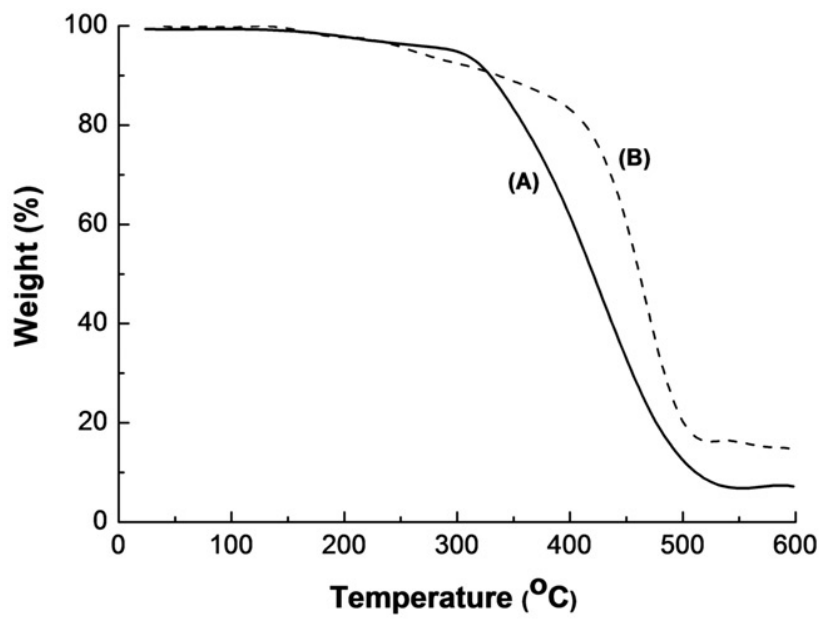

Figure 7. Thermal behaviour of EDA (curve A) and PPD (curve B) microcapsules. Samples were previously dried at $90^{\circ} \mathrm{C}$ during $12 \mathrm{~h}$.

was therefore attempted as a strategy to improve the microcapsule toughness. PPD:DETA molar ratios of 4:1, 3:2, 2:3 and 1:4 were tested. However, this did not eliminate the problem of microcapsule mechanical stability. The only clear effect is that the capsules
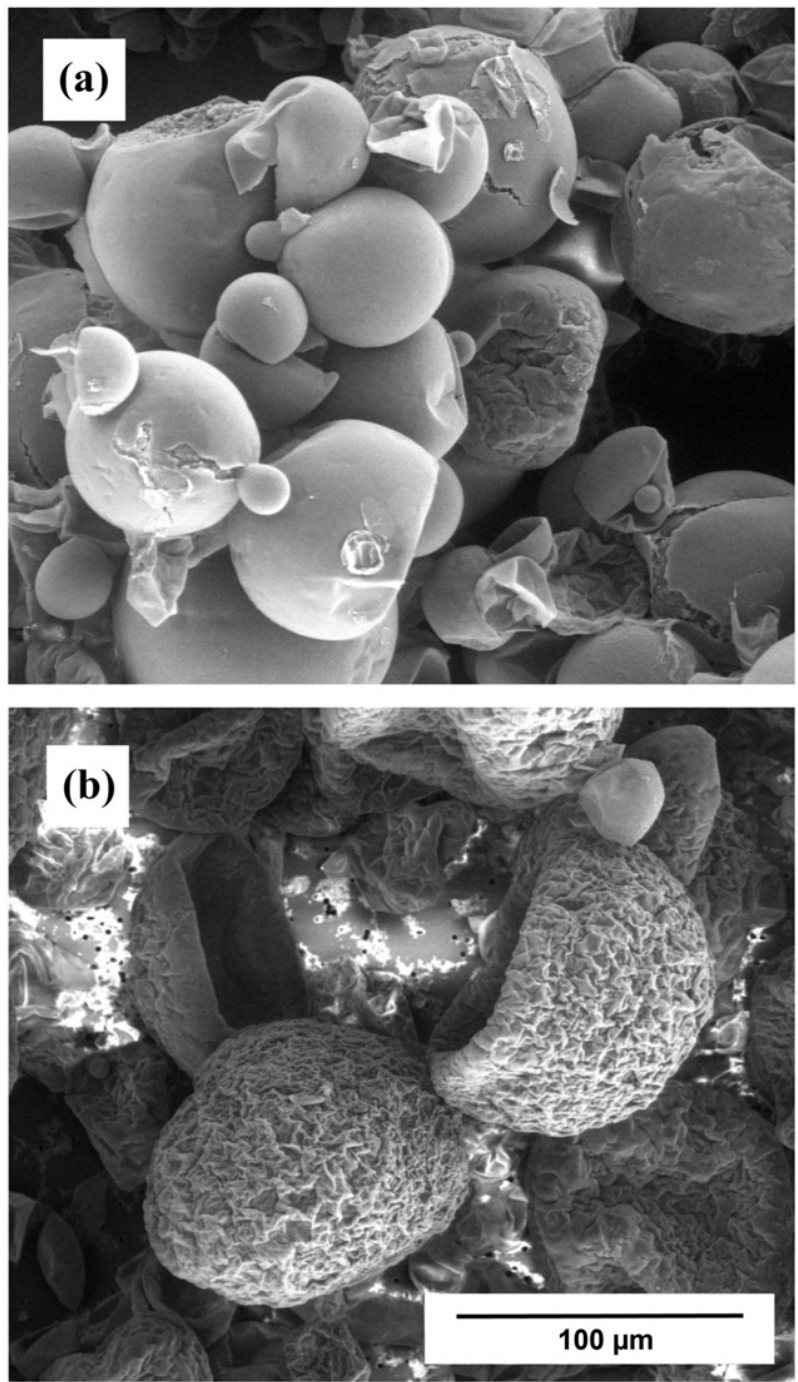

Figure 8. Scanning electron micrographs for microcapsules obtained with different proportions of PPD:DETA: 2:3 (a) and 1:4 (b).

tend to collapse ("deflate") instead of developing cracks upon drying, as seen in the SEM images of Figure 8, for PPD:DETA molar ratios of 2:3 and 1:4. Interestingly, for PPD:DETA above $2: 3$ the wall morphology progressively changes from smooth to wrinkled, as also seen in Figure 8 . This effect has been previously reported for polyamide capsules (Pascu et al., 2008; Salaün et al., 2009). The increase in cross-linker content makes the surface more uneven as a result of a more difficult diffusion of the monomers through the polymeric wall.

As an alternative strategy, addition of a plasticizer to the polyamide wall was attempted. Oleic acid was chosen due to its long aliphatic chain and carboxylic end group, which provides water insolubility together with a certain degree of amphiphilicity. This compound is a known oil-soluble surfactant with low HLB index (Schramm, 2000). This fact can potentiate its positioning at the water/oil interface, and facilitate incorporation of the aliphatic chain in the capsule wall during polymerization. Use of fatty acids, namely oleic acid and fatty acid derivatives as plasticizers for zein, chitosan and PVC has been discussed before in the literature (Srinivasa et al., 2007; Xu et al., 2012; Fenollar et al., 2013).

Figure 9 shows representative SEM images of microcapsules obtained when oleic acid was added to the organic phase at different percentages. In all cases the capsules maintained a smooth external surface and became remarkably intact as oleic acid concentration was increased. Table 3 presents the percentage 

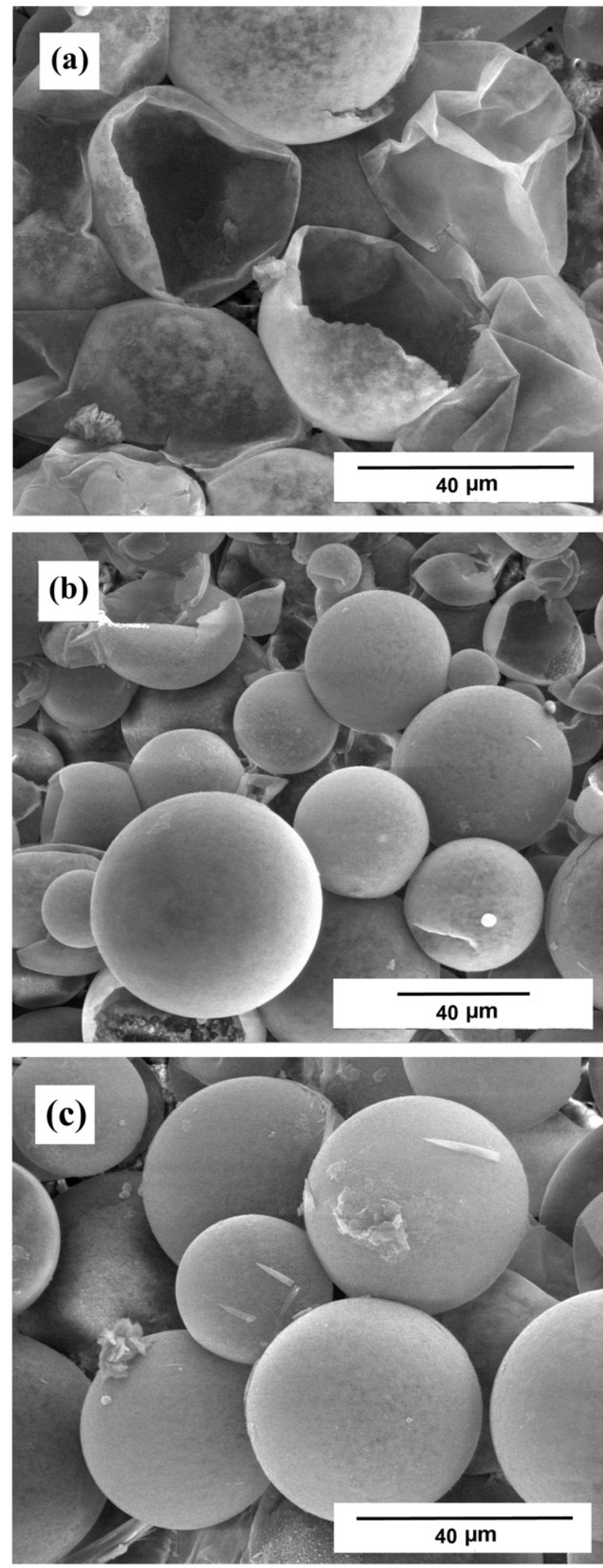

Figure 9. Scanning electron micrographs of microcapsules prepared with oleic acid addition to the organic phase: $1.8 \mathrm{wt} \%$ (a) $4.6 \mathrm{wt} . \%$ (b), and 9.2 wt.\% (c).

of damaged capsules for different oleic acid contents, based on SEM images analysis. At 9.2 wt.\% the majority of capsules are neither cracked nor collapsed (Figure 10a). This indicates that oleic acid addition yielded effective toughening of the polymeric walls. FTIR and NMR spectra were obtained for PPD microcapsules prepared with oleic acid addition. The capsules were crushed, washed and dried after synthesis for removal of the
Table 3. Fraction of damaged microcapsules as a function of oleic acid content.

\begin{tabular}{lc}
\hline $\begin{array}{l}\text { Oleic acid wt.\% in } \\
\text { organic phase }\end{array}$ & $\begin{array}{c}\text { Number of fractured capsules } \\
\text { (mean\% } \% \text { std. dev.) }\end{array}$ \\
\hline 0 & $>98.0 \%$ \\
1.8 & $94.3 \% \pm 1.4 \%$ \\
4.6 & $34.1 \% \pm 4.6 \%$ \\
9.2 & $15.7 \% \pm 4.5 \%$ \\
\hline
\end{tabular}
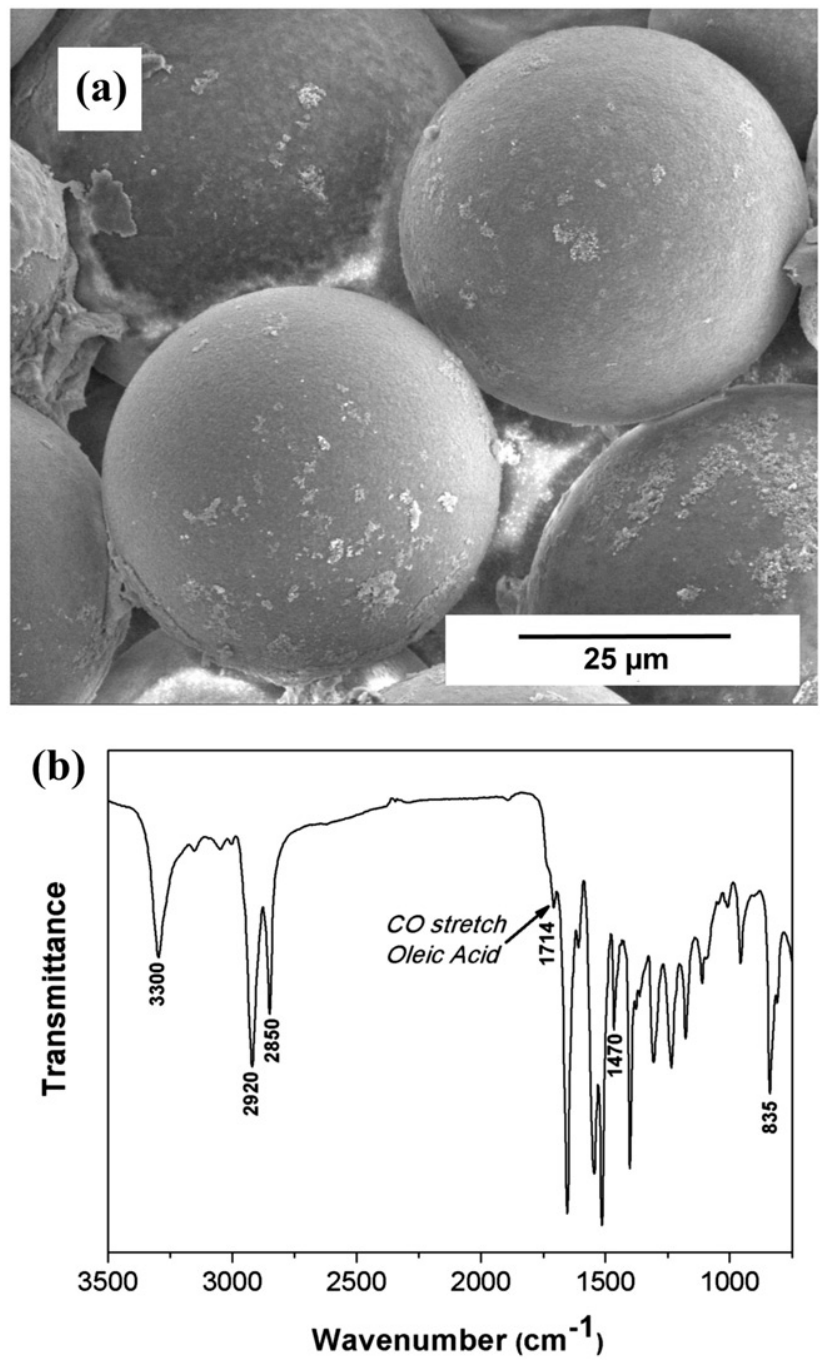

Figure 10. Scanning electron micrograph showing surface of microcapsules prepared with $9.2 \mathrm{wt}$ \% oleic acid addition to the organic phase (a). FTIR spectrum of PPD microcapsules containing oleic acid (b).

organic phase. The FTIR spectrum, in Figure 10(b), shows once again the characteristic bands that confirm formation of polyamide polymer. Moreover, a characteristic band associated with for carbonyl stretching in oleic acid is observed at about $1714 \mathrm{~cm}^{-1}$. In addition, the presence of oleic acid in the shell is also indicated by the increase in absorption intensities of the following bands, taking as reference the band assigned to $\mathrm{CH}$ deformation in para-disubstituted benzene $\left(835 \mathrm{~cm}^{-1}\right): \mathrm{CH}$ antisymmetric stretching band at $2920 \mathrm{~cm}^{-1}$ band, $\mathrm{CH}$ symmetric stretching band at $2850 \mathrm{~cm}^{-1}$ band, and $\mathrm{CH}_{2}$ bending vibration band at $1470 \mathrm{~cm}^{-1}$ band.

Figure 11 shows the ${ }^{1} \mathrm{H}-\mathrm{NMR}$ spectra of the shells obtained from PPD microcapsules in DMSO-d6, which were prepared with (curve A) and without (curve B) oleic acid in the organic phase. Solvent peaks appear at $\delta 2.50$ and $3.30 \mathrm{ppm}$, the latter being due 


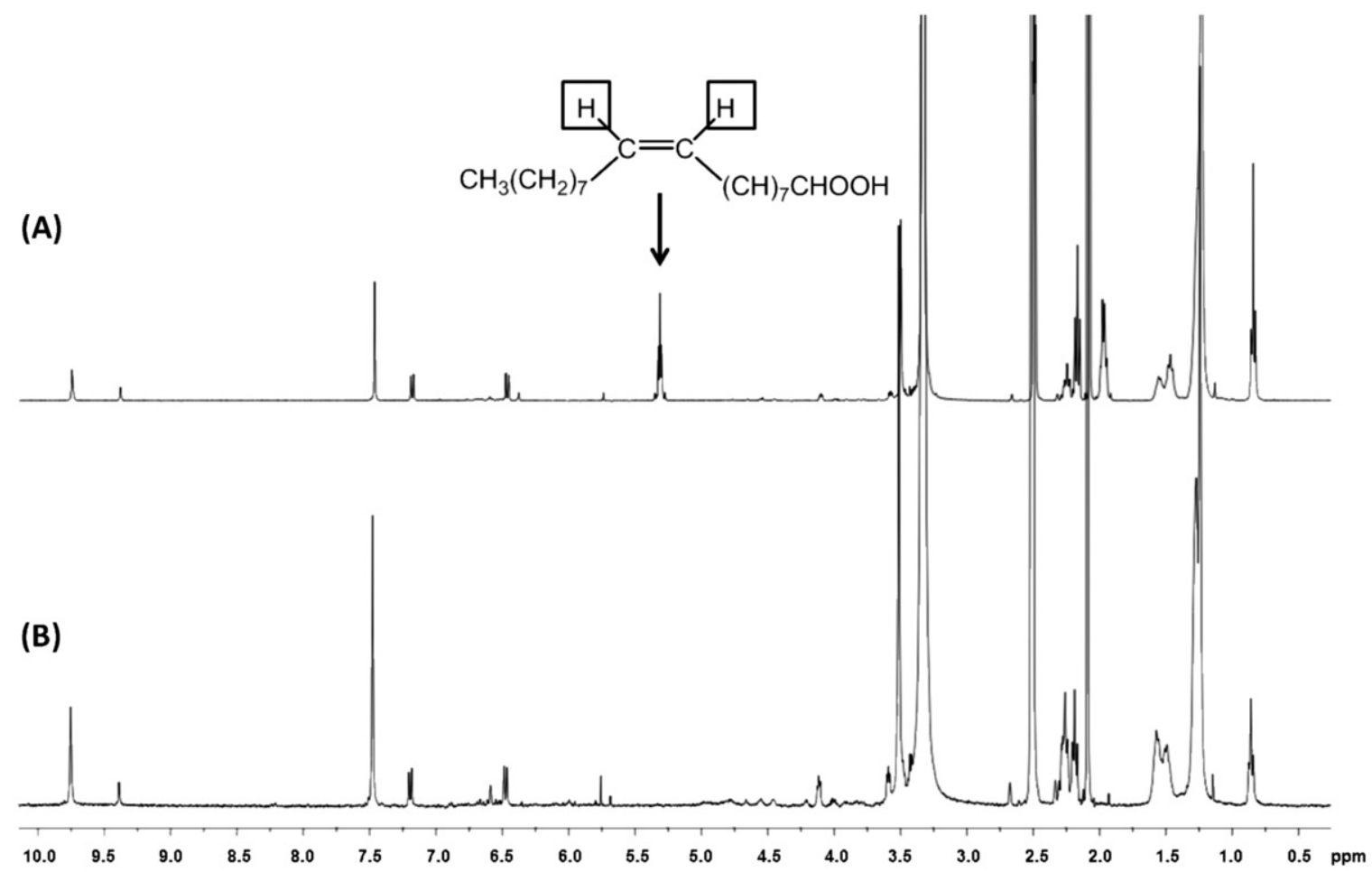

Figure 11. ${ }^{1}$ H-NMR spectra of PPD microcapsules: with (curve A) and without (curve B) oleic acid.

to contaminating water. In general, the NMR signal assignment confirms the polymeric structure expected for the reaction of PPD with SC. Both spectra exhibit characteristic singlets at $\delta 9.75 \mathrm{ppm}$, from $\mathrm{N} \underline{\mathrm{H}} \mathrm{CO}$ in amide bond, and at $\delta 7.47 \mathrm{ppm}$, corresponding to aromatic protons from $p$-phenylenediamine units in the polyamide. In addition, the signals in the ranges $\delta 1.4-1.6$ and $\delta 2.1-$ $2.3 \mathrm{ppm}$ can be attributed to the protons $\mathrm{CH}_{2} \mathrm{CH}_{2} \mathrm{CO}$ and $\mathrm{CH}_{2} \mathrm{CH}_{2} \mathrm{CO}$, respectively, from sebacoyl moieties. However, only spectrum A displays characteristic signals from oleic acid: a triplet at $\delta 5.30$, which is assigned to the two protons of the double bond, and a multiplet at $\delta 1.98$, from allylic hydrogens in $\mathrm{CH}_{2}-\mathrm{CH}=\mathrm{CH}-\mathrm{CH}_{2}$, proving its presence in the microcapsules. Interestingly, resonance signals corresponding to $\left(\mathrm{OCH}_{2} \mathbf{C H}_{2}\right)_{n} \mathrm{O}$ in surfactant Tween 20 (polyoxyethylene sorbitan laurate) appear at around $\delta 3.50 \mathrm{ppm}$, as well as a characteristic triplet at $\delta$ $0.85 \mathrm{ppm}$ from terminal $-\mathrm{CH}_{3}$ in laurate chain, demonstrating that this surfactant is also retained within the polymeric wall. In spectrum $\mathbf{A}$ the signal at $\delta 0.85 \mathrm{ppm}$ is intensified due to contribution from $-\mathrm{CH}_{3}$ in oleic acid.

Figure 12 presents the thermogravimetric curves obtained for non-encapsulated dodecane (curve A), PPD dried microcapsules at room temperature for $24 \mathrm{~h}$ (curve B), PPD microcapsules dried at $110^{\circ} \mathrm{C}$ for $24 \mathrm{~h}$ (curve $\mathrm{C}$ ), and PPD microcapsules dried in the same conditions but prepared with oleic acid addition (curve D). Non-encapsulated dodecane (curve A) shows significant evaporation rate above $50^{\circ} \mathrm{C}$, and at about $150{ }^{\circ} \mathrm{C}$ only a residual amount remains. The microcapsules dried at room temperature are expected to still contain dodecane, independently of being effectively encapsulated within the particle core or not. The corresponding thermogravimetric trace (curve B) shows a significant weight loss in the range $50-150^{\circ} \mathrm{C}$, which corresponds to the temperature range of free dodecane evaporation, as seen for curve A. The other weight loss step, above $400{ }^{\circ} \mathrm{C}$ is ascribed to decomposition of the polyamide, as previously discussed (Figure 7). This shows that, as expected for capsules with cracked shells, the oil was not effectively encapsulated. Concordantly, when the same microcapsules were dried at $110^{\circ} \mathrm{C}$ (curve $\mathrm{C}$ ), the majority of the weight loss occurs in the temperature range of

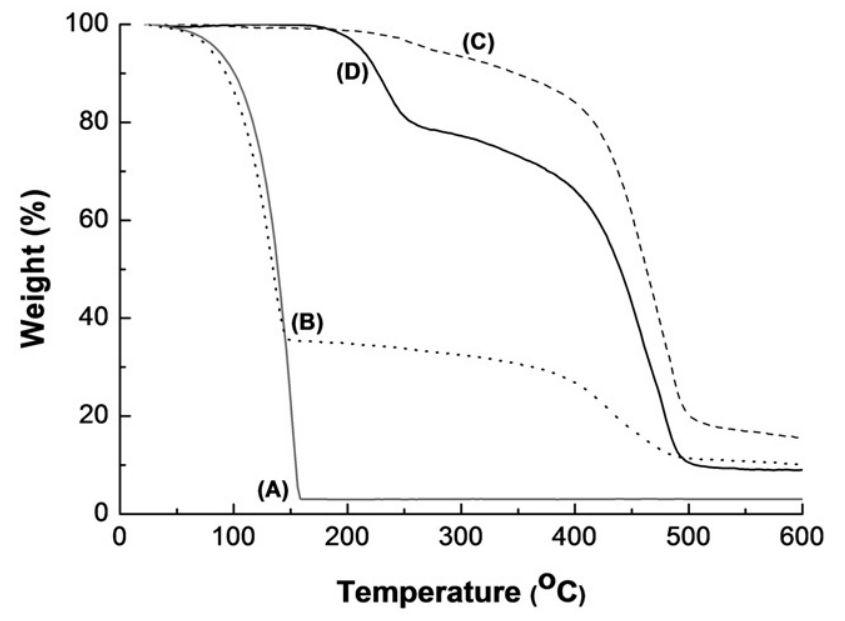

Figure 12. Thermal behaviour of non-encapsulated dodecane (curve A), PPD microcapsules dried at room temperature for $24 \mathrm{~h}$ (curve B), PPD microcapsules dried at $110^{\circ} \mathrm{C}$ for $24 \mathrm{~h}$ (curve C) and PPD microcapsules prepared with oleic acid and dried at $110^{\circ} \mathrm{C}$ for $24 \mathrm{~h}$ (curve D).

polyamide decomposition, indicating that the dodecane originally present has evaporated during drying. On the other hand, microcapsules prepared with oleic acid and dried at $110^{\circ} \mathrm{C}$ (curve D) show a first weight loss step at around $200^{\circ} \mathrm{C}$, indicating that dodecane, which has a boiling point of $216^{\circ} \mathrm{C}$, has only escaped after boiling conditions were attained and shell bursting occurred due to internal pressure increase. This corroborates the previous SEM analysis in showing that presence of oleic acid provides defect-free capsules.

Dodecane content, based on TGA trace D, is about 25 wt.\%. This value is relatively low and should be improved upon process optimization.

\section{Conclusion}

This work studied the microencapsulation of dodecane by interfacial polymerization of PPD and SC. The type of stabilizer 
used was shown to strongly influence the size, size distribution, and surface morphology of the microcapsules. The use of the aromatic diamine monomer yielded rigid polymeric walls that did not collapse after drying, unlike when using ethylenediamine. However, the capsules exhibited fragile fracture during processing. This limitation was effectively overcome by adding oleic acid as plasticizer. The slightly amphiphilic nature of the molecule allowed its positioning at the oil/water interface and subsequent incorporation in the polyamide wall during capsule formation. This strategy was much more effective than using a triamine cross-linker. Use of oleic acid as a capsule wall additive appears to be a straightforward and effective approach to improve the toughness of microcapsules produced by interfacial polymerization.

Oil encapsulation efficiency and capsule shell permeability have not been analysed and will be studied in future work, in the context of specific applications for special purpose oils.

\section{Declaration of interest}

This work was funded by FEDER funds through the Operational Programme for Competitiveness Factors (COMPETE), ON.2 - O Novo Norte - North Portugal Regional Operational Programme and National Funds through Foundation for Science and Technology (FCT) under the projects: PEst-C/EQB/UI0511, NORTE-07-0124-FEDER-000026 - RL1_ Energy and PTDC/CTM-NAN/119979/2010. The Bruker Avance III 400 spectrometer is part of the National NMR network and was purchased under the framework of the National Programme for Scientific Reequipment, REDE/1517/RMN/2005, with funds from POCI 2010 (FEDER) and (FCT). Joana R. Góis acknowledges FCT-MCTES for her PhD scholarship (SFRH/BD/69635/2010).

\section{References}

Arshady R. Preparation of microspheres and microcapsules by interfacial polycondensation techniques. J Microencapsul, 1989;6(1):13-28.

Benita S. (2005). Microencapsulation: Methods and industrial applications. Boca Raton, FL: CRC Press.

Berezkin AV, Khokhlov AR. Mathematical modeling of interfacial polycondensation. J Polym Sci Pol Phys, 2006;44(18):2698-724.

Caruso MM, Blaiszik BJ, Jin H, Schelkopf SR, Stradley DS, Sottos NR, White SR, Moore JS. Robust, double-walled microcapsules for selfhealing polymeric materials. ACS Appl Mater Interfaces, 2010; 2(4):1195-9.

Chen W, Liu X, Lee DW. Fabrication and characterization of microcapsules with polyamide-polyurea as hybrid shell. J Mater Sci, 2012; 47(4):2040-4.

Chuanjie F, Xiaodong Z. Preparation and barrier properties of the microcapsules added nanoclays in the wall. Polym Advan Technol, 2009;20(12):934-9.

Danicher L, Frère Y, Le Calvé A. Synthesis by interfacial polycondensation of polyamide capsules with various sizes. Characteristics and properties. Macromol Symp, 2000;151:387-92.

Dubey R. Microencapsulation technology and applications. Def Sci J, 2009;59(1):82-95.

Essawy H, Tauer K. Polyamide capsules via soft templating with oil drops -1. Morphological studies of the capsule wall. Colloid Polym Sci, 2010;288(3):317-31.

Essawy H, Tauer K. Polyamide capsules via soft templating with oil drops - 2. Subsequent radical polymerization of styrene. Colloid Polym Sci, 2011;289(2):121-32.
Fenollar O, Garcia-Sanoguera D, Sanchez-Nacher L, Boronat T, López J, Balart R. Mechanical and thermal properties of polyvinyl chloride plasticized with natural fatty acid esters. Polym Plast Technol Eng, 2013;52(8):761-7.

Freger V. Nanoscale heterogeneity of polyamide membranes formed by interfacial polymerization. Langmuir, 2003;19(11):4791-7.

Goel A, Rani N. Effect of PVP, PVA and POLE surfactants on the size of iridium nanoparticles. Open J Inorg Chem, 2012;2(3):67-73.

Hong K-J, Park S-M. Preparation and characterization of polyurea microcapsules with different diamines. Mater Res Bull, 1999; 34(6):963-69.

Latnikova A. 2012. Polymeric capsules for self-healing anticorrosion coatings, PhD Thesis. Max Planck Institut für Kolloid- und Grenzflächenforschung Abteilung, Potsdam, Germany.

Li Q, Mishra AK, Kim NH, Kuila T, Lau K-T, Lee JH. Effects of processing conditions of poly(methylmethacrylate) encapsulated liquid curing agent on the properties of self-healing composites. Compos Part B-Eng, 2013;49:6-15.

Mahabadi $\mathrm{H}, \mathrm{Ng} \mathrm{T}$, Tan $\mathrm{H}$. Interfacial/free radical polymerization microencapsulation: kinetics of particle formation. J Microencapsul, 1996;13(5):559-73.

Mittal V. (2013). Encapsulation nanotechnologies. Beverly, MA: John Wiley \& Sons.

Pascu O, Garcia-Valls R, Giamberini M. Interfacial polymerization of an epoxy resin and carboxylic acids for the synthesis of microcapsules. Polym Int 2008;57(8):995-1006.

Salaün F, Devaux E, Bourbigot S, Rumeau P. Influence of process parameters on microcapsules loaded with n-hexadecane prepared by in situ polymerization. Chem Eng J, 2009;155(1):457-65.

Scarfato P, Avallone E, Iannelli P, de Feo V, Acierno D. Synthesis and characterization of polyurea microcapsules containing essential oils with antigerminative activity. J Appl Polym Sci, 2007;105(6): 3568-77.

Schramm LL. (2000). Surfactants: Fundamentals and applications in the petroleum industry. Cambridge: Cambridge University Press.

Song Y, Sun P, Henry LL, Sun B. Mechanisms of structure and performance controlled thin film composite membrane formation via interfacial polymerization process. J Membrane Sci, 2005; 251(1):67-79.

Soto-Portas ML, Argillier JF, Mechin F, Zydowicz N. Preparation of oily core polyamide microcapsules via interfacial polycondensation. Polym Int, 2003;52(4):522-7.

Srinivasa P, Ramesh M, Tharanathan R. Effect of plasticizers and fatty acids on mechanical and permeability characteristics of chitosan films. Food Hydrocoll 2007;21(7):1113-22.

Wagh S, Dhumal S, Suresh A. An experimental study of polyurea membrane formation by interfacial polycondensation. J Membrane Sci, 2009;328(1):246-56.

Wittbecker EL, Morgan PW. Interfacial polycondensation. I J Polym Sci, 1996;34(4):521-9.

Wu G, An J, Sun D, Tang X, Xiang Y, Yang J. Robust microcapsules with polyurea/silica hybrid shell for one-part self-healing anticorrosion coatings. J Mater Chem A, 2014;2:11614-20.

$\mathrm{Xu}$ H, Chai Y, Zhang G. Synergistic effect of oleic acid and glycerol on zein film plasticization. J Agric Food Chem, 2012;60(40):10075-81.

Yan N, Zhang M, Ni P. Study on polyamide microcapsules containing oily liquids. J Microencapsul, 1994;11(4):365-72.

Zhang M, Ni P, Yan N. Effect of operation variables and monomers on the properties of polyamide microcapsules. J Microencapsul, 1995; 12(4):425-35.

Zydowicz N, Chaumont P, Soto-Portas M. Formation of aqueous core polyamide microcapsules obtained via interfacial polycondensation: Optimization of the membrane formation through $\mathrm{pH}$ control. J Membrane Sci, 2001;189(1):41-58. 